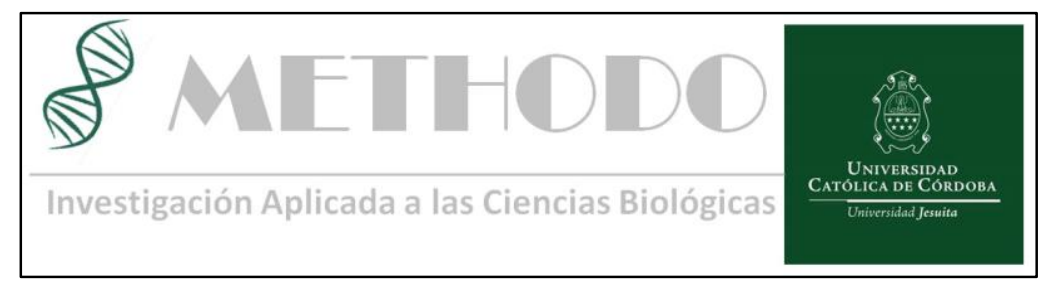

\title{
Criterios éticos y legales para la toma de decisiones en intervenciones relacionadas con nanotecnología.
}

\section{Ethical and legal criteria for decision making related to Nanotechnology.}

\author{
Diego Fonti, Dr. ${ }^{1}$, Cristian Baglini, Mgter. ${ }^{2}$, Florencia Irusta, Srta. ${ }^{3}$, Candelaria Valetti, Srta. ${ }^{3}$ \\ ${ }^{1}$ Investigador CONICET, Universidad Católica de Córdoba \\ ${ }^{2}$ Profesor Titular Cátedra de Odontología Legal y Forense, Carrera de Odontología, Facultad de Ciencias de la Salud, \\ Universidad Católica de Córdoba \\ ${ }^{3}$ Estudiante de la Carrera de Odontología, Facultad de Ciencias de la Salud, Universidad Católica de Córdoba \\ Carrera de Odontología, Facultad de Ciencias de la Salud, Universidad Católica de Córdoba, Libertad 1255 - Barrio \\ General Paz (5000) Córdoba
}

\section{Resumen:}

Las tecnociencias fundamentan su avance en la promesa de una mejor calidad de vida para el ser humano. Sin embargo, es necesario evitar el extremo del optimismo ignorante de los condicionantes económicos de las intervenciones tecno científicas, como también la ignorancia de las vulnerabilidades en juego. Una intervención capaz de obtener la legitimidad social implica que todos los involucrados puedan aceptar las consecuencias que se desprenden de ella. En el caso de las nanotecnologías, las intervenciones han de juzgarse según los criterios procedimentales y según la Declaración Universal de Bioética y Derechos Humanos, que al mismo tiempo podrán regir las nuevas reglamentaciones, normas y leyes que las intervenciones requieran, particularmente en previsión de sus resultados futuros personales, sociales y ambientales.

\section{Abstract:}

Technosciences base their innovations on the promise of a better quality of life for human beeings. However, it is essential to avoid the innocent optimism of economic conditionants of technological and scientific interventions as well as to ignore the hazards of these attempts. A socially legitimate intervention implies that every participant involved would be able to accept the consequences that derive from it. As regards to Nanotechnology, such interventions should be analyzed under the light of their procedures criteria and the Universal Declaration of Bioethics and Human Rights, that will also rule a new legislation later required for each development, forthseeing personal, social and environmental outcomes.

\section{Introducción:}

Una de las características de la Modernidad ha sido el avance de las tecnociencias sobre casi todos los aspectos de la vida. Mientras que antes de la Modernidad el conocimiento tecnocientífico encontraba en la naturaleza un criterio moral para sus intervenciones, la crítica de Hume a ese vínculo como "falacia naturalista" permitió la separación entre ambos campos, científico y ético. A partir de esta "liberación", la tecnociencia intervino en múltiples ámbitos de un modo cada vez menos regulado, y ha adquirido además la característica de una automatización anónima, con procesos que exceden a menudo la voluntad y el control de las personas. Los resultados de este proceso alcanzan niveles macro $\mathbf{y}$ micro, con intervenciones cada vez más transformadoras en ambos extremos. Ante este proceso surge la pregunta sobre las normas que han de orientar dichas transformaciones (a nivel de investigación y clínica) en el ámbito de la salud, además de la pregunta fundamental por la justificación de esas 
normas, que al mismo tiempo evite tanto recaer en los criterios de un período pre-moderno como en el relativismo, que finalmente es cómplice de las imposiciones del poder. Así es que en un mundo de "extraños morales", con multiplicidad de valores y fundamentos éticos diversos, se hace necesario encontrar una base moral legítima compartida y universal, capaz de entender el problema, abordarlo y formular criterios de cuidado y responsabilidad para evitar que esas transformaciones afecten capacidades humanas y la dignidad de las personas, y al mismo tiempo para garantizar que los conocimientos y técnicas legítimas sean accesibles a modo de derechos universales.

Nos proponemos elaborar, en primer lugar, una exposición estructural de las características de nuestra era tecnocientífica en general, y de las particularidades de la nanotecnología. En segundo lugar, abordaremos a partir de la ética procedimental de Habermas las condiciones de posibilidad para una toma de decisiones legítima en nuestra época. ${ }^{1}$ A continuación, expondremos las normas legales actuales - y los aspectos donde se evidencia una carencia de las mismas - en torno a este campo. Y finalmente, a modo de conclusión, propondremos una serie de indicaciones prácticas para abordar posibles conflictos o dilemas en este ámbito, que tengan en cuenta los aspectos éticos y legales expuestos.

\section{Tecnociencia y nanotecnología: características estructurales}

Desde las narraciones míticas la técnica se ha mostrado como ambigua, necesaria y peligrosa. La vida humana es imposible sin técnica, pero la técnica misma -y sus consecuencias- ha puesto a la vida humana y la naturaleza toda en riesgo. Heidegger ha mostrado cómo la técnica es una "estructura de emplazamiento", es decir, un modo que fuerza las cosas y hace salir lo oculto en ellas, "trae-ahí-delante" las potencias inherentes a ellas. ${ }^{2}$ La ciencia no es una perspectiva "objetiva" desde la cual juzgar a la técnica sino la convalidación de su modo de producir el emplazamiento del mundo. Para Heidegger la verdad es aletheia, o sea desocultamiento. Como todo des ocultamiento, se trata de un proceso que abre y oculta al mismo tiempo. En el caso de la tecnología y su convalidación bajo la estructura de la ciencia, se trata de toda una era que entiende la relación con el mundo, las cosas y las personas desde esa perspectiva de manipulación con vistas al manejo instrumental y previsible de su funcionamiento y consecuencias.

Para Heidegger el peligro de este modo de "hacer salir lo oculto" se debe a la posibilidad de impedir que salga a la luz lo más originario del hombre y su relación con la totalidad de lo que existe. Por supuesto que hoy discutiríamos qué es eso "original" del hombre, pero más allá de esas discusiones sí parece posible estar de acuerdo que la técnica opera en el ser humano (y la cultura, y la naturaleza) una reducción notable. Las intervenciones nanotecnológicas lo muestran de un modo particular.

Se denomina "nanotecnología" al campo disciplinar que se encarga del estudio, creación, diseño, síntesis, identificación, manipulación y aplicación de materiales, aparatos y sistemas a través del control de la materia en dimensiones cercanas al intervalo de 1-100 nanómetros, así como de la exploración de fenómenos y propiedades de la materia a dicha escala. En el campo de la odontología, la nanotecnología alude al tipo de intervenciones destinadas a mejorar la relación que existe entre las nanoestructuras de las piezas dentarias y los microorganismos colonizadores. Estas intervenciones abren una inmensa variedad de oportunidades y posibilidades en diferentes especialidades clínicas. Por ejemplo, las nanopartículas de plata que se están utilizando como alternativa a los agentes para obturaciones dentales en operatoria son relevantes ya que presentan propiedades de antidesgaste, antibacterianas y antifúngica y ser capaz de combatir Staphylococcusaureus, Encherichiacoli, Enterococcusfaecalis y Candidaalbicans. Por su parte, en lo que se refiere a la regeneración del esmalte y la dentina, en la fabricación de órganos dentarios in vitro encontramos la combinación de bioingeniería tisular, junto con el desarrollo de nanopartículas genéticamente diseñadas y de nanopartículas que sean biomiméticas con los tejidos mineralizados. Además, en ortodoncia se están aplicando nanopartículas que controlan la señalización del dolor y que aumentan la ramificación de los nervios al utilizar nanoesferas rellenas de factores que inducen a la regeneración del tejido nervioso. Finalmente, el área de implantología es en la actualidad la especialidad con más desarrollo a través de un material denominado nanohueso, que imita muy de cerca la estructura y composición de los huesos reales, lo que convertirá a los implantes artificiales de titanio en un material del pasado. Podemos pensar que en un futuro cercano será clave su uso en el diagnóstico de enfermedades de alto impacto como el cáncer de mama, ovario y páncreas, la enfermedad de Alzheimer, el HIV, la diabetes y osteoporosis.

Frente a estos avances, las directrices científicotecnológicas hacia el año 2020 se encaminan a la transformación de la odontología, mediante el uso de nanosistemas para el tratamiento de diferentes padecimientos. Sin embargo, se debe hacer énfasis en este tipo de intervenciones podría olvidar que 
existen todavía factores sociales, así como dilemas éticos, que son relevantes para una regulación relativa a establecer tratamientos en humanos y que se tienen que afrontar para que la nanotecnología pueda incorporarse en la odontología de manera completa y con legitimidad moral y normatividad legal. Es decir, se trata de un campo donde, siguiendo la exposición de Heidegger, se pone a disposición un ámbito en el cual se emplaza a las partículas más pequeñas de la materia dominable para su uso activo. La pregunta que surge ante esta dinámica es sobre los fines, límites, riesgos y accesibilidad de dicha intervención, es decir, sobre la legitimación de la misma en un sentido amplio. Dicha legitimación debe formularse tanto en clave moral como legal.

\section{Legitimidad y validez en la discusión ética}

Frente a este devenir histórico no cabe el romanticismo ni la creencia inocente que el progreso en el dominio del mundo conllevará también un progreso moral. Es por eso que atentos a la necesidad de criterios de evaluación de las propuestas, conviene recurrir a la ética procedimental de Habermas. ${ }^{1}$ Es una ética discursiva, en tanto sostiene que en la discusión que busca acuerdos normativos vinculantes, con respeto de un procedimiento que les legitime, es donde se hallará la legitimidad de los resultados. Es preciso también agregar a esta ética una noción actualizada del principio de responsabilidad de Jonas, en tanto la convivencia con personas de convicciones diversas a las nuestras no puede impedir la formulación de criterios regulativos comunes que eviten daños inadmisibles respecto de quienes tomamos las decisiones o quienes se verán afectados por ellas. ${ }^{3}$

Habermas (1985) formula criterios ineludibles para un acuerdo legítimo en condiciones de simetría y libertad de coacciones: En cualquier discusión que busque consensos que resuelvan el curso de acción y las normas sobre un tema conflictivo, toda persona involucrada debe poder expresar su posición, sus deseos y necesidades. Todos pueden problematizar toda afirmación. A nadie puede impedírsele ejercer estos derechos, so pena de tergiversar el sentido mismo de la comunicación que busque acordar con otros sobre un conflicto. Además, una vez que se llega a una decisión ha de constatarse su validez, esto es, si los afectados pueden aceptar las consecuencias que para cada uno se desprendan de la misma. Sucede que sólo pueden pretender validez las normas que No existe financiación, conflicto de intereses, ni patrocinante en la realización de este caso clínico. se dan como resultado de un discurso práctico entre hablantes.
Es preciso agregar a este procedimiento de Habermas un criterio relevante de Jonas (1995). Los efectos transformadores de nuestras acciones muestran que las éticas anteriores, que se basaban en la decisión individual y en un punto concreto del tiempo, se equivocaban en tanto desconocían los efectos futuros y globales de nuestras acciones. De allí que el principio de responsabilidad indica que debemos obrar de tal manera que los efectos de nuestras acciones sean compatibles con la permanencia de una existencia humana auténtica con todas las condiciones del patrimonio natural y cultural - en la tierra. Ninguna intervención humana - ni siquiera aquellas con "buenas intenciones" - puede ir contra la integridad misma de las condiciones de posibilidad de la vida en su interrelación. La vulnerabilidad del ser humano y de la naturaleza se ha convertido en un criterio bioético relevante, en tanto buena parte del daño que se puede hacer al otro ser humano actual y al otro ser humano futuro no es solamente un daño de causalidad directa, sino también debido a las múltiples acciones e intervenciones cuyos resultados se ramifican en efectos poco atendidos e intergeneracionales.

Por supuesto que en cualquier discusión sobre normas puede haber manipulación de la información, amenaza, coacción, pero esas serían todas formas ilegítimas de búsqueda de acuerdos. En cambio, un acuerdo que tenga en cuenta a los participantes y su situación futura, y al mismo tiempo que tenga en cuenta la preservación de la integridad del patrimonio natural, especialmente en relación con las condiciones de la humanidad, tendría un tipo de validez capaz de juzgar moralmente las intervenciones propuestas con un criterio de legitimidad válido en condiciones contemporáneas.

\section{Normas legales y nanotecnología}

En nuestro país es la Constitución Nacional el cimiento que sirve de base de todo el ordenamiento jurídico, junto con los Tratados Sobre los Derechos Humanos. Sobre esta base se asientan las diferentes Leyes Nacionales y Provinciales, y los Decretos que brindan diferentes tipos de derechos sobre los ciudadanos. También está a la base de las Resoluciones sobre la Investigación en Salud, qué se pueden constatar en el Atlas Federal de Legislación Sanitaria de la República Argentina, que se fueron sumando a las pautas éticas internacionales, para la investigación biomédica en seres humanos, formuladas por la Organización Panamericana de la Salud y la Organización Mundial de la Salud. Estas normativas señalan cómo aplicar en forma eficaz los principios éticos que deben regir la ejecución de la investigación biomédica en seres humanos, especialmente en los

Revista Methodo: Investigación Aplicada a las Ciencias Biológicas. Universidad Católica de Córdoba. 
países en desarrollo, dadas sus circunstancias socioeconómicas leyes y reglamentos y sus disposiciones ejecutivas y administrativas. Estos modelos de reconocimiento pueden relacionarse directa o indirectamente con el tema de la investigación clínica (por ejemplo, la libertad individual, el derecho de ser informado, de no recibir tratos inhumanos o degradantes, de recibir atención de salud, etc.) y la futura accesibilidad al conocimiento y productos investigados. Así también, se establece el deber que se le asigna al mismo Estado, de preservar dichos derechos. Según un trabajo de Investigación de la Universidad de Chile dirigida por el Doctor Roberto Mancini Rueda consultor del programa Regional de bioética de la O.P.S. y la O.M.S.,permitió establecer la existencia de comités de ética nacionales en 8 países y la ausencia total de normativas y comités de ética en 10 países (48\% de la muestra). Por último, respecto de la evaluación de protocolos de investigación, en 6 países $(29 \%)$ no se cumple ese requisito y en los restantes se aprecian serias deficiencias en la evaluación. Este análisis está siendo complementado con una evaluación de lo que ocurre en las instancias académicas y en las publicaciones científicas, con resultados preliminares bastante negativos. ${ }^{4}$ Esta realidad guarda directa relación con el exiguo desarrollo de normativas sobre investigación y experimentación en seres humanos, en la gran mayoría de los países de América Latina y el Caribe. Sólo podría señalarse a Brasil y Cuba como excepciones. En estos países se cuenta con un reglamento específico que les ha dado muy buenos resultados, y ha sido sometido a revisión continua y con el respaldo de las más altas autoridades sanitarias y políticas.

Solamente en los últimos tres años, en Argentina, Chile, Ecuador y México, se han desarrollado iniciativas similares, pero su breve aplicación no permite aún tener conclusiones sobre la mejoría de los protocolos de investigación en el plano ético. El estudio referenciado muestra cómo en Chile, luego de un largo proceso de análisis multidisciplinario y con participación de numerosas instancias relacionadas, se editó una Norma Ministerial que modifica algunos aspectos del Código Sanitario, del Reglamento de Servicios de Salud y del Instituto de Salud Pública, introduciendo la revisión ética de los protocolos de investigación en que se efectúan ensayos clínicos con medicamentos en seres humanos. Estas referencias muestran cómo es indispensable controlar jurídicamente los trabajos clínicos en Argentina, con un criterio ético y bioético que surja de la interacción reflexiva de los propios científicos y otros actores de la comunidad académica y social, y que sea inducida progresivamente a todos sus pares, para evitar así que las variables económicas que hay atrás de estas investigaciones clínicas tengan primacía por encima del bien social de la salud. Además, tales normativas incluyen también el tipo de protección necesaria frente a los riesgos de la comercialización en la investigación científica.

Finalmente, en el ámbito legal se desprende una consecuencia importante del carácter que la propia Constitución Nacional otorga a las Declaraciones relativas a Derechos Humanos. En 2005 la ONU emitió su Declaración Universal de Bioética y Derechos Humanos, que Argentina ha firmado y por lo tanto se considera vinculante tanto para la Constitución como para todas las leyes derivadas. ${ }^{5}$ Esta Declaración asume los principios de protección, reconocimiento de la vulnerabilidad y los requisitos de cuidado del patrimonio natural y cultural de la humanidad, así como también la disponibilidad de los conocimientos y técnicas capaces de proveer el bien de la salud de modo universal.

\section{Conclusión:}

Es necesario evitar el extremo del optimismo ignorante de los condicionantes económicos de las intervenciones tecno científicas, como también la ignorancia de la vulnerabilidad es en juego. Una intervención capaz de obtener la legitimidad social implica que todos los involucrados

puedan aceptar las consecuencias que se desprenden de ella. En el caso de las nanotecnologías, las intervenciones han de juzgar se según los criterios procedimentales y según la Declaración Universal de Bioética y Derechos Humanos, que al mismo tiempo podrán regir las nuevas reglamentaciones, normas y leyes que las intervenciones requieran, particularmente en previsión de sus resultados futuros personales, sociales y ambientales.

\section{Bibliografía:}

1. Habermas, J. Conciencia moral y acción comunicativa. Barcelona: Península; 1985.

2. Heidegger, M. "La pregunta por la técnica". Conferencias y artículos. Barcelona: Odos; 1994.

3. Jonas, H. El principio de responsabilidad. Barcelona: Herder; 1995.

4. Mancini Rueda, R., Lolas Stepke, F. "Evaluación bioética de trabajos de investigación en seres humanos publicados en América Latina y el Caribe", Acta Bioethica 2001; año VII, nº 1, 159-169. 
5. Organización de Naciones Unidas. Declaración Universal de Bioética y Derechos Humanos; 2005 (disponible http://portal.unesco.org/es/ev.phpURL_ID=31058\&URL_DO=DO_TOPIC\&U RL_SECTION=201.html)Epilepsyehav (2016),

http://dx.doi.org/10.1016/j.yebeh.2016.11.01 $\underline{2}$

\section{Palabras claves:}

TECNO CIENCIAS, BIOÉTICA, DERECHOS HUMANOS

Keywords:

TECHNOSCIENCES, BIOETHICS, HUMAN RIGHTS.

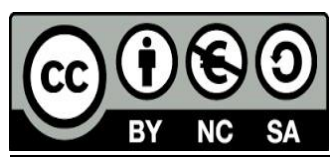

